\title{
'Unsticking' a colloidal glass, and sticking it again
}

\author{
W C K Poon ${ }^{1}$, K N Pham, S U Egelhaaf and P N Pusey \\ School of Physics, The University of Edinburgh, Mayfield Road, Edinburgh EH9 3JZ, UK \\ E-mail: w.poon@ed.ac.uk
}

Received 16 October 2002

Published 16 December 2002

Online at stacks.iop.org/JPhysCM/15/S269

\begin{abstract}
We study glass formation in hard spheres with short-range attraction. The system consists of nearly-hard-sphere polymethylmethacrylate particles and non-adsorbing random-coil polystyrene which induced a depletion attraction between the particles. The experiments reveal a re-entrant glass transition and two qualitatively distinct glassy states. Dynamic light scattering, covering eleven orders of magnitude in time, gives insight into the kinds of particle motion responsible for these observations. The possible relevance of our results to generic issues, such as the distinction between fragile and strong glass formers, the nature of the underlying 'free energy landscape', and the relative importance of temperature and pressure, is discussed.
\end{abstract}

(Some figures in this article are in colour only in the electronic version)

\section{Introduction}

Understanding glasses is an outstanding challenge facing 21 st century physics, with relevance extending well beyond physics to materials science and biology [1]. In this enterprise, simple model systems hold an important place. Experimentally, one of the simplest model systems is a suspension of sterically stabilized polymethylmethacrylate (PMMA) particles. In suitable hydrocarbon solvents, these particles behave as almost-perfect hard spheres. Computer simulations predict that for $N$ hard spheres of radius $R$ confined to volume $V$ (so that the volume fraction $\left.\phi=(4 / 3) \pi R^{3} N / V\right)$, the lowest free energy state at $\phi<\phi_{F}=0.494$ is a fluid-amorphous arrangement in which the particles are individually free to explore the whole available volume, given time. For $0.545=\phi_{M}<\phi<\pi / \sqrt{18} \approx 0.74$, however, the equilibrium state is crystalline. Experiments using PMMA particles [4] confirmed this picture, except that homogeneous crystallization was observed to cease at $\phi>\phi_{g} \approx 0.58$. This has been interpreted as a glass transition: amorphous arrangements of particles becoming 'stuck', and not rearranging into crystals.

\footnotetext{
1 Author to whom any correspondence should be addressed.
} 
The glass transition in sterically-stabilized PMMA suspensions has been studied in considerable detail by the dynamic light scattering (DLS) technique, which probes the decay of spontaneously occurring density fluctuations at various wavelengths. The DLS data can be fitted remarkably well by mode-coupling theory (MCT) predictions concerning the hardsphere glass transition [5]. The picture that emerges from both of these methodologies is that of 'caging'. In a dense hard-sphere fluid, each particle spends significant time being 'caged' by its neighbours. At long enough times, the identity of this cage of neighbours changes. We can say that the cage dissolves, and the particle can undergo long-range diffusion. But each particle, as well as being caged, is also part of the cages of its neighbours. Such coupling becomes catastrophic at $\phi_{g}$ : the cooperative rearrangements necessary for cage dissolution become impossible, and a glass results. This effect shows up in DLS [5] as a two-staged decay of the intermediate-scattering function, $f(q, t)$, at scattering vector $q$-decorrelation of the scattered light occurs at short time by particles 'rattling' in their cages, and, fully, at longer times by cages opening. In the glass, the timescale for the longer-time process diverges, giving a non-zero $^{2} f(q, \infty)$. In MCT [6], where the sole input is the static structure factor, $S(q)$, the key 'glass-forming' modes are those round the peak of $S(q)$ —cages in reciprocal space.

In this paper, we present an experimental study of what happens to the hard-sphere glass transition when a short-range interparticle attraction is turned on. We do this by using a wellcharacterized model system [7]: random-coil polystyrene (radius of gyration $r_{g}=17 \mathrm{~nm}$ ) mixed with PMMA particles $(R=202 \mathrm{~nm})$ dispersed in cis-decalin. Exclusion of the (non-adsorbing) polymer coils from the region between the surfaces of two nearby particles creates an unbalanced osmotic pressure pushing the particles together. The range of this 'depletion' attraction is essentially the size of a polymer coil, a dimensionless estimate of which is $\xi=r_{g} / R$. The topology of the equilibrium phase diagram is controlled by $\xi$. For the case studied in this paper, where $\xi \sim 0.08$, the addition of polymer merely expands the region of fluid-crystal coexistence, which occurs at $\phi_{F}<\phi<\phi_{M}$ in pure hard spheres.

\section{Observation of re-entrant glass transition}

The non-equilibrium behaviour of colloid-polymer mixtures at $\xi \sim 0.1$ and low colloid volume fractions (say, $\phi \leqslant 0.2$ ) has been studied before $[8,9]$. Here we concentrate on the region $\phi \geqslant 0.3$. Mixtures with different values of $\phi$ and polymer concentrations $\left(c_{p}\right)$ were prepared, homogenized by prolonged tumbling, and then left undisturbed for observation. Because of the size of the particles, colloidal crystals can be easily detected by the naked eye as iridescent specks. Our observations are shown in figure 1. According to theory [11], adding polymer at small $\xi$ expands the region of fluid-crystal coexistence, and this is what we observe (diamonds, figure 1). Again, consistent with theory, samples at higher $\phi$ were fully crystalline (inverted triangles). For samples with the highest polymer and/or colloid concentrations, however, no crystallization was observed within a period of weeks to months (filled squares and circles), even though equilibrium statistical mechanics predicts either fluid-crystal coexistence (for the filled squares) or full crystallization (for the filled circles).

Consider a sample sequence $\mathrm{A}-\mathrm{G}$, figure 1 , at $\phi \approx 0.6$. In thermodynamic equilibrium, all of these samples should crystallize [11]. Sample A without any polymer was a glass. Sample C with $\sim 1.4 \mathrm{mg} \mathrm{cm}^{-3}$ of polymer, however, completely crystallized, i.e. $\phi_{g}$ has shifted to higher volume fractions. Cessation of crystallization was again observed at polymer concentration around $2.4 \mathrm{mg} \mathrm{cm}^{-3}$. From the behaviour of all the samples we find that the non-crystallization line is re-entrant. For pure hard spheres, crystallization ceases at the point when $f(q, \infty)$ first

\footnotetext{
$2 \infty=$ 'times long compared to the measurement window'. See comments on 'ageing', below.
} 


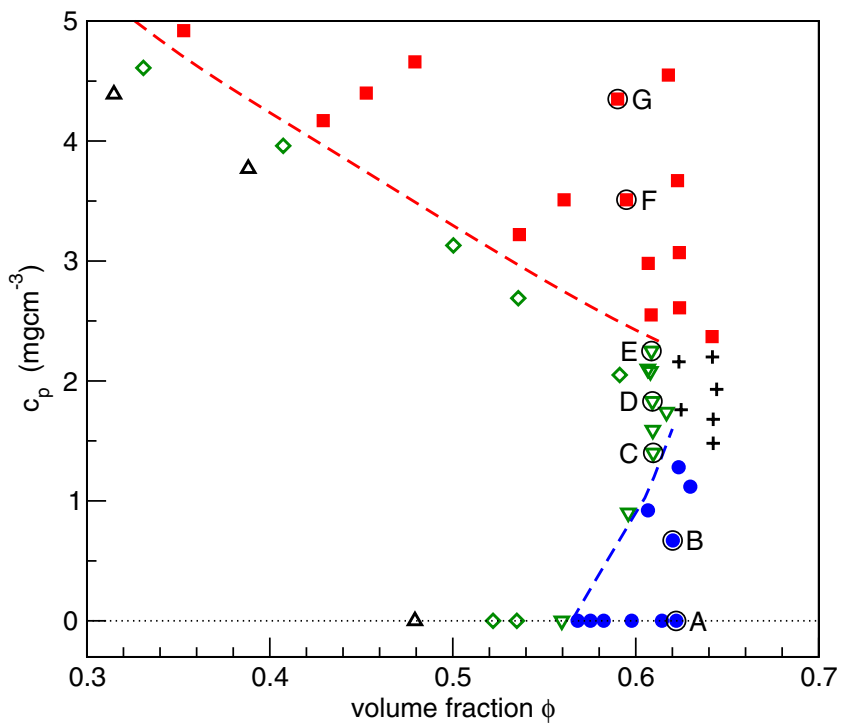

Figure 1. Equilibrium and non-equilibrium behaviour of a colloid-polymer mixture of $\xi=0.08$. Open symbols represent samples that reached thermal equilibrium: fluid (triangles), fluid-crystal coexistence (diamonds), and fully crystallized (inverted triangles). Other samples did not reach thermal equilibrium: some showed characteristics of hard-sphere glass (circles), some showed those of attraction-driven glass (squares), and some showed both (plus signs). (After a period of weeks to months, non-equilibrium samples showed different signatures of sedimentation. Repulsive glassy samples (circles) showed very small amounts of tiny crystals on the meniscus with no clear supernatant. The attractive glasses (squares) showed tiny amounts of clear supernatant without any crystals. The other samples (crosses) had a tiny amount of clear supernatant with very small amounts of tiny crystals just below the sedimentation boundary.) Dashed curves are guides to the eye showing the observed boundary where crystallization ceased. DLS data for circled samples labelled A-G are shown in figure 3. (This diagram is a fuller version of the one presented in [10], with extra data points at high $\phi$.)

becomes non-zero $[4,5]$. If this coincidence still holds when polymer is added, then figure 1 tells us that adding 'stickiness' to hard spheres leads to a re-entrant glass transition.

Computer simulation, MCT, and preliminary DLS [10,12] findings suggest the following heuristic picture; figure 2. In the 'repulsion-dominated' hard-sphere glass, particles are caged by their neighbours. A little short-range attraction clusters the particles in the cage and opens up holes, ultimately melting the glass. Increasing the attraction further leads to an 'attractiondominated' glass_-particles stick to their neighbours with long-lived bonds.

\section{Dynamic light scattering studies}

To elucidate further this re-entrant glass transition and the nature of the two glassy states involved, detailed DLS studies were carried out on a sequence of samples with approximately constant $\phi$ and increasing $c_{p}$ (A-G, figure 1). Because our samples are somewhat turbid, two-colour DLS was used to access $f(q, t)$ [13]. Results at $q R=1.5$ over a time window of nearly eleven decades [14] are shown in figure 3.

A quantitative discussion of these results will be presented elsewhere [16]. Here we point out some striking qualitative features. Sample A shows the classic behaviour of a hard-sphere glass. The decay process setting in at $t \sim 10^{-3} \mathrm{~s}$ is due to particles rattling in their cages. 

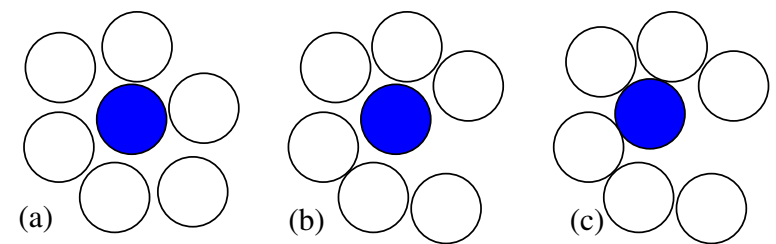

Figure 2. A heuristic picture of the re-entrant glass transition. (a) At low attraction, the shaded particle is caged by its neighbours. (b) Adding short-range attraction clusters the particles that form the cage, thus opening up holes, and eventually melting the glass. (c) At high enough attraction, there are long-lived bonds between all touching particles and the whole system arrests into another glassy state.
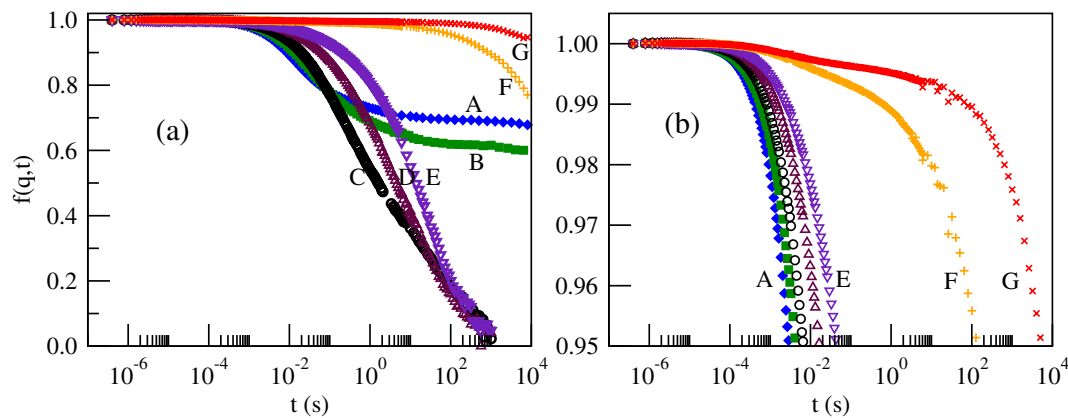

Figure 3. (a) Normalized collective dynamic structure factors $f(q, t)$ obtained from DLS at $q R=1.5$ for samples marked $\mathrm{A}-\mathrm{G}$ in figure 1 . (b) The same quantities are re-plotted with an expanded vertical axis. Very high incipient plateaus of $f(q, t)$ for samples $\mathrm{F}$ and $\mathrm{G}$ can be recognized. The initial decays slow down monotonically from A to G. (Similar observations have been made for microgel particle + non-adsorbing polymer mixtures [15].)

These cages never 'open', however, and $f(q, \infty)$ plateaus at $\sim 0.7$. Adding a small amount of polymer, sample B, appears to slow down the 'rattling' — see especially figure 3(b) — but weakens the confining effect of the cage: $f(q, \infty)$ drops to $\sim 0.6$. Further addition of polymer should give rise to samples that ultimately crystallize; figure 1 . As expected, $f(q, t)$ for sample $\mathrm{C}$, measured while it was a metastable fluid (i.e. before crystallization), decays completely to zero. The shape of this correlation function is, however, surprising. The $f(q, t)$ for a pure hard-sphere fluid at $\phi>0.56$ is expected to decay in two steps: associated respectively with rattling in the cage and cage opening (see, e.g., data in [5]). Such a two-stage decay is almost invisible in the $f(q, t)$ of sample $\mathrm{C}(\phi=0.61)$ - there is only a slight slowing down of the rate of decay at $t \sim 3 \mathrm{~s}$, just visible as a 'kink' in the data. Addition of polymer produces further metastable fluid samples, D and E, with completely decaying $f(q, t)$ s that do not even show such a 'kink', but only progressive overall slowing down with increasing $c_{p}$. For sample F, $f(q, t)$ begins to develop a plateau at $\sim 0.993$ over approximately one decade in time before decaying further. This plateau moves higher for sample $\mathrm{G}$.

Note that samples $\mathrm{F}$ and $\mathrm{G}$ showed ageing: $f(q, t)$ depended on the time $t_{W}$ waited before starting the measurement (data not shown; see [16]). In particular, the decay moves out to longer times as $t_{W}$ increases. This is behaviour expected of glasses [17]; metastable fluids are not expected to 'age' in this manner. 
We explain the data in figure 3 in the following manner ${ }^{3}$. In a dense hard-sphere fluid, particles rattle freely in the cage of their neighbours (slowed down slightly relatively to free particle diffusion only by hydrodynamic interactions [18]). By the time the cage opens/dissolves, a particle will have explored the interior of the cage many times. In fact, it is this separation of timescales that permits us to talk in terms of a 'mean-field' cage picture. At $\phi_{g}$, the cage becomes permanent.

Addition of polymer has two consequences. First, as already noted, it clusters the particles in a cage and, eventually, leads to cage opening and melting of the glass; cf figure 2(b). At the same time, however, the short-range interparticle attraction also slows down the 'rattling' motion, because a particle gets trapped in potential wells of varying depths. This slowing down is monotonic with respect to $c_{p}$ (or, equivalently, deepening interparticle attraction); figure 3(b). At the point where the repulsion-dominated glass melts $\left(c_{p} \sim 1 \mathrm{mg} \mathrm{cm}^{-3}\right.$ for $\phi \sim 0.6$; figure 1), the cage opening time and the timescale for an elementary step in the rattling motion become comparable: $f(q, t)$ for sample $\mathrm{C}$ shows only a hint of a two-step decay. Thereafter, it is no longer meaningful to talk of a cage picture. The dynamics of the particles is controlled by a single characteristic timescale, that involved in escaping the attractive traps of their neighbours: $f(q, t)$ for samples D and E show no sign of two-step decay at all. Divergence of this timescale finally leads to the attraction-dominated glass. The particles are now trapped within attraction wells of width $\xi \sim 0.08$. This correlates with the very high plateaus in the $f(q, t)$ s of samples $\mathrm{F}$ and $\mathrm{G}$.

These observations are consistent with predictions of MCT [19]. Detailed fitting of the data according to the predictions of this theory will be presented elsewhere [16]. Nevertheless, one aspect of the picture presented here is supported by a heuristic calculation. We approximate the depletion potential by a linear ramp from $-U$ at contact to zero at a distance $\Delta$ away. A particle with diffusion coefficient $D$ bonded to $z$ neighbours will escape with a characteristic time [20]

$$
\tau \sim \frac{\Delta^{2}}{D} \frac{\mathrm{e}^{\beta z U}}{(\beta z U)^{2}},
$$

where $\beta=\left(k_{B} T\right)^{-1}$. Taking $D$ to be $\sim 10 \%$ of the free particle value, $z=3$, and $\beta U \sim 3$ for sample $\mathrm{C}$ (calculated using standard depletion formulae), we can reproduce the observed single characteristic time of $\sim 1 \mathrm{~s}$ for sample $\mathrm{C}$. The increase of $\beta U$ to $\sim 5$ upon moving to sample $\mathrm{E}$ together with a decrease to $z \sim 2$ reproduces the observed order-of-magnitude slowing down of the single characteristic time of this sample relative to that of sample C.

\section{Conclusions: colloidal and atomic/molecular glasses}

To conclude, we briefly discuss the possible relevance of our results to glass studies in general. Hard spheres are athermal, but temperature plays an important role in atomic and molecular systems. Nevertheless, the hard-sphere glass transition is thought to resemble at least qualitatively certain aspects of the glass transition in 'fragile' glass formers in atomic and molecular systems. Adding attraction to hard spheres may be thought to bring colloidal systems one step closer to their atomic and molecular counterparts. However, the attraction considered in this work is short range — 10\% of the hard core: we are dealing with 'sticky hard spheres'. This is very much unlike the case for atomic and molecular systems, where the range of attractive interactions is comparable to the size of the hard cores. Now, MCT predicts [19] that the distinction between repulsion-dominated and attraction-dominated glasses disappears long before this limit is reached, so we cannot expect any direct mapping of phenomenology. Nevertheless, sticky hard-sphere glasses may still raise certain generic issues.

\footnotetext{
3 The following heuristic discussion is for single particles, and does not strictly apply to data in figure 3 for $q R=1.5$.
} 


\subsection{Fragile-strong}

Atomic and molecular glasses are classified as 'fragile' and 'strong' according to the way the viscosity diverges as the glass transition is approached (from the liquid side) [2]. The viscosity of strong glass formers diverges according to an Arrhenius law (i.e. exponentially), while that of fragile glass formers does not. Hard spheres are then fragile. We may speculate that attraction-dominated glasses are 'strong' in this sense-because there is an energy scale (set by the attractive well depth), we may expect an 'activated' (i.e. Arrhenius) viscosity. Strong and fragile glasses can also be distinguished by their underlying 'energy landscapes' [3]. Confocal imaging of our two glassy states may also shed light on the issue.

\subsection{Enthalpic-entropic}

Consider now an issue that has been controversial in the atomic and molecular glass literature for some time [21]: is the glass transition mainly temperature or volume/density driven? Stated differently, the issue is whether we have mainly an energetic or entropic effect. A recent study of triphenyl phosphite [21] found that around ambient pressure, energy (or temperature) is the dominant variable, although the authors suggested that at elevated pressure, density could become dominant. In this language, our model system is driven to structural arrest in the repulsion-dominated regime by volume/density/entropy, while the attraction-dominated glass is arrested largely because of energy/temperature. This analogy may be worth pursuing.

\subsection{Various theoretical approaches}

MCT has been found to give significant insight into the glass transition in hard spheres without as well as with a short-range attraction. Much of the physical basis for its success, however, remains to be elucidated. Other approaches are possible, but have been less used to discuss colloidal glasses to date. Some of these may give insight into sticky hard-sphere glasses. Energy landscapes [3] have already been alluded to. This approach is at least somewhat related to the random-trap model [22]. Note that the environment of each particle, crucial to both approaches, can in principle be deduced from confocal microscopy observation if the interparticle potential is known.

Finally, we note that the melting of the hard-sphere glass by adding a little stickiness can perhaps be fruitfully discussed in terms of 'free volume' [23]. Rather than focusing our attention on the particles, we may instead say that attraction creates a clustering of free volume, thus enabling large-scale motions. Again, the free volume is a quantity that is in principle available from direct imaging.

\section{Acknowledgments}

It is a pleasure to thank Dr M Fuchs for many fruitful discussions. KNP holds a UK ORS Award. Partial financial support for this project came from EPSRC grant GR/M92560.

\section{References}

[1] See the wide-ranging survey articles (particularly [2] and [3]) in 1995 Science 2671887

[2] Angell C A 1995 Science 2671924

[3] Stillinger F H 1995 Science 2671935

[4] Pusey P N and van Megen W 1986 Nature 320340 
[5] van Megen W and Underwood S M 1994 Phys. Rev. E 494206

[6] Bengtzelius U, Götze W and Sjölander A 1984 J. Phys. C: Solid State Phys. 175915

[7] Poon W C K 2002 J. Phys.: Condens. Matter 14 R859

[8] Poon W C K, Pirie A D and Pusey P N 1995 Faraday Discuss. 10165

[9] Poon W C K et al 1999 Faraday Discuss. 112143

[10] Pham K N et al 2002 Science 296104

[11] Lekkerkerker H N W et al 1992 Europhys. Lett. 20559

[12] Puertas A, Fuchs M and Cates M E 2002 Phys. Rev. Lett. 88098301

[13] Segrè P N et al 1995 J. Mod. Opt. 421929

[14] Pham K N, Egelhaaf S U and Pusey P N 2003 in preparation

[15] Eckert T and Bartsch E 2002 Faraday Discuss. 123 at press

[16] Pham K N, Fuchs M, Egelhaaf S U, Pusey P N and Poon W C K 2003 in preparation

[17] Bouchaud J-P 1999 Soft and Fragile Matter, Nonequilibrium Dynamics, Metastability and Flows (Scottish Universities Summer School in Physics, 2000) ed M E Cates and M R Evans (Bristol: Institute of Physics Publishing)

[18] van Megen W et al 1998 Phys. Rev. E 586073

[19] Dawson K et al 2000 Phys. Rev. E 63011401 and references therein

[20] Hanggi P, Talkner P and Borkovec M 1990 Rev. Mod. Phys. 62251

[21] Ferrer M L et al 1998 J. Chem. Phys. 1098010

[22] Monthus C and Bouchaud J-P 1996 J. Phys. A: Math. Gen. 293847

[23] Cohen M H and Grest G S 1979 Phys. Rev. B 201077 